\title{
nPIST: A Novel Actin Binding Protein of trans-Golgi Network
}

Swagata Das ${ }^{*}$, Priyanka Dutta* ${ }^{1}$, Mohit Mazumder ${ }^{2}$, Soma Seal ${ }^{1}$, Kheerthana Duraivelan ${ }^{3}$,

Dibyendu Samanta ${ }^{3}$, Samudrala Gourinath ${ }^{2}$ and Sankar Maiti ${ }^{\# 1}$

1 Department of Biological Sciences, Indian Institute of Science Education and Research,

Kolkata. Nadia, West Bengal, INDIA.

2 School of Life Sciences, Jawaharlal Nehru University, New Delhi, INDIA

3 School of Bioscience, Indian Institute of Technology Kharagpur, Kharagpur, West Bengal, INDIA

*Contributed equally

\# To whom correspondence should be addressed: Sankar Maiti, IISER-Kolkata, India

E-mail: spm@iiserkol.ac.in

Keywords: nPIST, trans-Golgi network, WH2-domain, actin, vesicular trafficking

\section{Abbreviations:}

G-actin, globular actin; F-actin, filamentous actin; FADE, Fast Atomic Density Evaluation;

KFC2, Knowledge-based FADE and Contacts; CC1, Coiled-coil helix 1; CC2, Coiled-coil helix 2. 


\begin{abstract}
:
nPIST is the neuronal isoform of PIST, a trans-golgi associated protein involved in major modulation of vesicular trafficking. nPIST interacts with glutamate delta2 receptor (GluR $\delta 2)$ in Purkinje cells. Our study shows nPIST as a novel actin binding protein. Our structure based sequence analysis shows nPIST contains one WH2-like domain. Further our experimental analysis illustrates that fragment of nPIST consisting of WH2-like domain binds to actin. Moreover it was found that nPIST contains several regions involved in interaction with actin. The binding of nPIST to actin through multiple actin binding regions facilitated actin filament stabilization in vitro. In vivo, nPIST localized actin in perinuclear region as a blotch when ectopically expressed.
\end{abstract}

\title{
Introduction:
}

PIST (PDZ domain protein interacting specifically with TC10) also known as GOPC (Golgiassociated PDZ and coiled-coil motif-containing protein) is primarily discovered as TC10 Rho GTPase interacting protein (Neudauer et al.; 2001). It is also known as CAL [cystic fibrosis transmembrane conductance regulator (CFTR)-associated ligand] (Cheng et al.; 2002, and 2004) and FIG (Fused in Glioblastoma) (Charest et al.; 2003, and Cheng et al.; 2004). PIST is expressed in all type of mammalian tissues whereas its neuronal isoform known as nPIST is exclusively expressed in mammalian whole brain preferentially in parallel fiber synapses of cerebellar Purkinje cells, hippocampus and cerebral cortex (Cuadra et al.; 2004, Yue et al.; 2002, Yao et al.; 2001, and Chen et al.; 2012). It has two N-terminal coiled-coil domains and one Cterminal postsynaptic density-95/discslarge/zonula occludens-1 (PDZ) domain (Neudauer et al.; 2001, Cuadra et al.; 2004, Yue et al.; 2002, and Yao et al.; 2001). The second coiled-coil domain is required for its golgi localization and interaction with Golgi resident proteins (Charest et al.; 2001, and Hicks and Machamer; 2005) whereas the PDZ domain is known to interact with several plasma membrane destined proteins. In the past decade, PIST has been well established as a key Golgi resident candidate for regulation of intracellular sorting and trafficking of plasma membrane targeted proteins.

In initial studies, PIST emerged as interacting partner of several transmembrane proteins having wide array of functional aspect. Interestingly, the mode of action of PIST for different proteins 
differs from one another. The plasma membrane expression of Rhotekin, mGluR5a (Metabotropic Glutamate Receptor subtype 5a), and Frizzled5 is enhanced by their interaction with PIST (Ito et al.; 2006, Cheng et al.; 2010, and Yao et al.; 2001). On the other hand, PIST restricts plasma trafficking of diverse types of membrane spanning proteins. Among all these proteins, some are ion channels like CFTR, CIC3B (ClC Chloride Channel 3B) (Cheng et al.; 2002, 2004, and Gentzsch et al.; 2003); some are receptors like SSRT5 (Somatostatin Receptor subtype 5), mGluR1a (Metabotropic Glutamate Receptor subtype 1a), B1AR (B1-Adrenergic Receptor), B2AR (B2-Adrenergic Receptor), CRFR1 (Corticotropin-releasing Factor Receptor 1) (Wente et al.; 2005, Zhang et al.; 2008, He et al.; 2004, Yang et al.; 2015, and Hammad et.; 2015); cell adhesion molecules cadherin23 (Xu et al.; 2010), and protocadherin15 (Nie et al.; 2016); tight junction proteins claudin1 and 2 (Lu et at.; 2015); ABC transporter mrp2 (Li et al.; 2017) etc. The Golgi retention of most of these proteins results in their degradation (Cheng et al.; 2010, 2013, and Lu et al.; 2015) but in contrast, B1AR is stabilized and mGluR5a is salvaged from polyubiquitination through interaction with PIST (He et al.; 2004, and Cheng et al.; 2010).

Remarkably, PIST is not only involved in golgi mediated trafficking but might also play major role in golgi morphology and maintenance. In PIST knockdown mice, spermatogenesis gets completely hampered and develops several cellular process dysfunctions which cumulatively results in globozoospermia. Among these cellular deteriorations, the major event is malformation of acrosomal cap in spermatids. Other than occurrence of vesicular or fragmented acrosome; nuclear malfunction and abnormal arrangement of mitochondria also takes place. Moreover, in $\mathrm{PIST}^{-/-}$condition seminiferous tubule loses its normal F-actin filament distribution. Actin filaments fail to surround the adjacent spermatids and get accumulated in basal region (Yao et $a l . ; 2002$, and Ito et al.; 2004). The neuronal isoform nPIST contains eight amino acids extra (151-158 aa) in the second coiled-coil domain. nPIST is primarily known to interact with Glutamate $\delta 2$ Receptor (GluR $\delta 2$ ) (Yue et al.; 2002). Like its non-neuronal isoform, nPIST is also involved in vesicular trafficking regulation. The clustering of AMPA receptor GluR1 to synapse is synchronized by interaction between AMPAR-interacting protein Stargazin and nPIST (Cuadra et al.; 2004).

The indispensable role of both PIST and nPIST in diverse cellular and physiological activities implies that they might have direct or indirect interaction with actin cytoskeleton. Functional 
characterization of nPIST is not studied in details like that of its non-neuronal isoform. In this study we have characterized nPIST as a novel actin binding protein. As nPIST have mostly coiled-coil structure, we searched for WASP homology 2 domain (WH2) in nPIST by multiple sequence alignment with WH2 domains of other well characterized actin binding proteins. The alignment detected one WH2-like domain having conserved amino acids which are characteristic feature of WH2 domain of several actin binding proteins (Paunola et al.; 2002). Our study suggested that nPIST is able to interact with actin through its multiple actin binding regions and stabilizes filamentous actin in in vitro condition. Further it was observed that nPIST gets localized to perinuclear region inside HEK293 cells through its N-teriminal coiled-coil region and causes abnormal accumulation of cellular F-actin co-localizing with it.

\section{Results:}

\section{nPIST, a trans-Golgi associated protein have putative WH2 domain:}

We build several structural models of nPIST protein using Swiss model and modeller. The models shown in these studies were energy minimized after modelling to get a lower energy and more stable conformation. The full-length nPIST is composed of 463 amino acid residues with two domains; N-terminal coiled-coil region and the C-terminal contain PDZ signaling domain. Based on the sequence information we built various models of $\mathrm{N}$ and $\mathrm{C}$ terminal of nPIST from closely related structures based on the template search with sequence identity of more than 30 percent sequence identity. The top scoring templates with known domains are shown in (Fig. 1A) along with the sequence coverage at the top. The model of coiled coil domain region $\left(34^{\text {th }}-194^{\text {th }}\right.$ aa, and $74^{\text {th }}-204^{\text {th }}$ aa) is shown in (Fig. 1B) and the PDZ domain region models with identified WH2 domain $\left(213^{\text {th }}-374^{\text {th }}\right.$ aa, $201^{\text {st }}-370^{\text {th }}$ aa, and $215^{\text {th }}-368^{\text {th }}$ aa) is shown in (Fig. 1C). The structural model revealed that N-terminal coiled-coil region as predicted (secondary structure) was highly helical compared to extreme C-terminal PDZ domain region which was comparatively disordered and composed of many loop regions. The central helix formed a hinge giving flexibility to the molecule to move. The full length structure based sequence analysis revealed that nPIST contains WH2 domain within the coiled-coil region. The WH2 domain was identified on the basis of sequence conservation, evolutionary function and structure of the domain. Based on structural model we performed sequence based structural alignment of nPIST with all the available crystal structures of $\mathrm{WH} 2$ domain. The alignment clearly suggested that 
coiled-coil domain is present in N-terminus of nPIST and the probable WH2 domain is present around $230^{\text {th }}$ to $250^{\text {th }}$ amino acids (Fig. 1D). nPIST WH2 domain folded exactly same as seen in various structures co-crystallized with G-actin (Dominguez and Holmes, 2011), suggesting that the identified domain might work similarly. To understand more about the structural details of actin binding we modeled nPIST WH2 domain with G-actin using rosetta Flexpepdock server. The docking results suggested that nPIST WH2 domain binds adequately with G-actin (Fig. 1E). The mode of binding is similar to VopL actin complex (Rebowski et al.; 2010). The complex was then subjected to further analysis for identification of key residues responsible for actin binding using KFC-2 (Knowledge-based FADE and Contacts) server. The server analyzed several chemical and physical features surrounding an interface residue and predicted the classification of the residue using a model trained on prior experimental data. The final complex obtained after simulations was used as the input for KFC2. The analysis was done for WH2 domain in which TRP-238, GLN-240, LEU-241, ILE-245 were predicted as binding hotspots (Table 1). Figure1F showed location of hotspots at the binding interface of G-actin predicted by KFC2 server.

\section{Full length nPIST has actin binding ability:}

The full length nPIST $\left(1^{\text {st }}-463^{\text {rd }}\right.$ aa) (Fig. 2A) was cloned in pET28a vector and expressed in $E$. coli B121-DE3-RP strain. nPIST was purified as N-terminal 6-His tagged protein (Fig. 2B). Purified nPIST showed molecular size of $~ 60$ KDa on SDS PAGE; however the theoretical size of nPIST is $54 \mathrm{KDa}$ including 6 His-tag flanking region. To resolve this, we had sequenced our cloned nPIST ORF and found that its sequence is identical with the nPIST sequence from database with ID BAC27058.1 from EMBL-EBI. From computational analysis it was predicted that nPIST contains a putative WH2 domain (Fig. 1C and D). To inquire whether nPIST also acts as other $\mathrm{WH} 2$ domain containing actin binding proteins, we performed F-actin co-sedimentation assay with full length nPIST. In the reaction set up, increasing concentrations $(0.5 \mu \mathrm{M}, 1 \mu \mathrm{M}, 2$ $\mu \mathrm{M}, 3 \mu \mathrm{M}, 4 \mu \mathrm{M}$, and $5 \mu \mathrm{M}$ ) of nPIST was incubated with $5 \mu \mathrm{M}$ of F-actin. Only $5 \mu \mathrm{M}$ nPIST containing reaction was used as negative control. After 310 x $1000 \mathrm{~g}$ centrifugation, the supernatant and pellet fractions were run in SDS PAGE. The coomassie stained SDS PAGE showed that the band intensity of full length nPIST in pellet fraction gradually increased when added to the reaction in increasing concentration (Fig. 2C). In protein control; there was very 
negligible amount of nPIST in pellet fraction which was much less than that of the reaction having $5 \mu \mathrm{M}$ of nPIST incubated with actin. This result illustrated that full length nPIST got cosedimented with F-actin in the pellet fraction in a concentration dependent manner. This revealed full length nPIST was able to bind actin like other actin binding proteins (Shimada et al.; 2004).

\section{nPIST has high affinity for F-actin, but not G-actin:}

The specific binding of nPIST to actin molecules was determined by Surface Plasmon Resonance (SPR) analysis which monitors molecular interaction in real time condition. Varying concentrations of F-actin and G-actin solutions were passed over purified nPIST protein (immobilized onto a series S CM5 sensor chip) and binding was detected by changes in RUs over time. The association time of both monomeric and filamentous actin to nPIST indicated rapid intermolecular interaction. However, the affinity of F-actin to nPIST was much higher than that of G-actin molecules (Fig. 3, and S1E). In case of F-actin, the equilibrium dissociation constant $\left(K_{\mathrm{D}}\right)$ was calculated to be $\sim 13.3 \mathrm{nM}$ that illustrates high molecular affinity, with $k_{\mathrm{a}}=$ $9.25 \times 10^{4} \mathrm{M}^{-1} \mathrm{~s}^{-1}$ and $k_{\mathrm{d}}=1.23 \times 10^{-3} \mathrm{~s}^{-1}$ (Table 2). On the other hand, G-actin did not show stable interaction with nPIST (Fig. S1E).

\section{WH2 domain containing fragment of nPIST enough for actin interaction:}

To check whether WH2 domain containing nPIST fragment was solely binding to actin, we had dissected nPIST into two fragments. For that, full length nPIST was sub-cloned in two parts; Nterminal coiled coil region having $\mathrm{WH} 2$ domain $\left(\mathrm{CC}-\mathrm{WH} 21^{\text {st }}-267^{\text {th }}\right.$ aa) and C-terminal PDZ domain region devoid of $\mathrm{WH} 2$ domain $\left(\Delta \mathrm{WH} 2,268^{\text {th }}-463^{\text {rd }}\right.$ aa, $($ Fig. $4 \mathrm{~A})$. The $C C-\mathrm{WH} 2$ and $\Delta \mathrm{WH} 2$ fragments were purified as 6-His tagged protein (Fig. 4B). The $\Delta \mathrm{WH} 2$ fragment was visualized in SDS PAGE at $\sim 30 \mathrm{KDa}$ (Fig. 4B, lane 2) but the theoretical size of the protein is 24.5 KDa with tag. We had sequenced our construct and like full length nPIST, $\Delta \mathrm{WH} 2$ fragment also showed sequence identity with the database sequence. F-actin co-sedimentation assay with these fragments showed $\mathrm{CC}-\mathrm{WH} 2$ fragment was co-sedimented along with F-actin in a concentration dependent manner in pellet fraction when $4 \mu \mathrm{M}, 8 \mu \mathrm{M}$ and $12 \mu \mathrm{M}$ of $\mathrm{CC}-\mathrm{WH} 2$ fragment was used for the reactions (Fig. 4C). Surprisingly, the $\Delta \mathrm{WH} 2$ fragment was also visible in pellet fraction with sedimented actin in increasing amount (Fig. 4D). In both the cases, there were very negligible amount of $\mathrm{CC}-\mathrm{WH} 2$ and $\triangle \mathrm{WH} 2$ protein in the pellet fraction of the 
respective protein control reactions. From this result, it was palpable that $\mathrm{CC}-\mathrm{WH} 2$ as well as $\Delta \mathrm{WH} 2$ fragment of nPIST had the ability to bind to actin.

\section{nPIST interacts with actin through multiple regions:}

The above result hinted that nPIST might have more sites engaged in interaction with actin other than the putative WH2 domain (Fig. 1B). To identify other possible actin binding regions, full length nPIST was truncated into smaller stretches and these fragments were sub-cloned in pET28a vector. The fragments containing first coiled-coil domain $\left(\mathrm{CC} 1,1^{\text {st }}-138^{\text {th }}\right.$ aa), second coiled-coil domain $\left(\mathrm{CC} 2,140^{\text {th }}-210^{\text {th }}\right.$ aa), modeled WH2 domain $\left(\mathrm{WH} 2,200^{\text {th }}-289^{\text {th }}\right.$ aa) and only PDZ domain (PDZ, 285 $5^{\text {th }}-463^{\text {rd }}$ aa) were taken into consideration (Fig. 5A). The Nterminal 6-His tagged CC1 fragment $\left(1^{\text {st }}-138^{\text {th }}\right.$ aa) (Fig. 5B), CC2 fragment $\left(140^{\text {th }}-210^{\text {th }}\right.$ aa) (Fig. 5C), WH2 fragment $\left(200^{\text {th }}-289^{\text {th }}\right.$ aa) (Fig. 5D), and PDZ fragment $\left(285^{\text {th }}-463^{\text {rd }}\right.$ aa) (Fig. 5E) were overexpressed in E. coli BL21-DE3-RP cells. Corresponding nPIST fragments were purified and run in SDS PAGE (Fig. 5B, 5C, 5D, and 5E). Like full length nPIST and $\triangle \mathrm{WH} 2$ fragment $\left(268^{\text {th }}-463^{\text {rd }}\right.$ aa $)$, the PDZ fragment $\left(285^{\text {th }}-463^{\text {rd }}\right.$ aa $)$ also occurred in higher molecular size $(\sim 27 \mathrm{KDa})$ than its theoretical value $22.7 \mathrm{KDa}$ with 6-His tag in SDS-PAGE (Fig $5 \mathrm{E})$. The WH2 fragment $\left(200^{\text {th }}-289^{\text {th }}\right.$ aa) protein was small in size and very degradation prone. So, it could not be further purified by gel-filtration chromatography to get rid of non-specific bands (Fig. 5D). The CC1, CC2, WH2 and PDZ fragments were subjected to F-actin cosedimentation assay, the supernatant and pellet fractions separated and run in SDS PAGE. The co-sedimentation assay gel image of CC1 fragment showed that the band intensity of CC1 in pellet fraction was present in rising order whereas the pellet fraction of only $12 \mu \mathrm{M} \mathrm{CC} 1$ negative control reaction had little $\mathrm{CC} 1$ fragment protein (Fig. 5F). The co-sedimentation assay of $\mathrm{CC} 2$ fragment (Fig. 5G) and WH2 fragment (Fig. 5H) exhibited their presence in pellet fraction in escalating amount when incubated with $5 \mu \mathrm{M}$ F-actin in concentration dependent manner. On contrary, the pellet fractions of the only CC2 fragment and WH2 fragment reaction had very negligible concentration of corresponding constructs (Fig. 5G and $\mathrm{H}$ ). We also found that PDZ fragment $\left(285^{\text {th }}-463^{\text {rd }}\right.$ aa) got co-sedimented with actin in pellet fraction in negligible amount (Fig. 5I). Here to be noted that one actin binding region is lying between WH2 domain and PDZ domain $\left(268^{\text {th }}-284^{\text {th }}\right.$ aa) which can be indicated from rapid reduction in affinity for 
actin of PDZ fragment (Fig. 5I) upon deletion of these amino acids from $\Delta \mathrm{WH} 2$ fragment ( Fig. 4D) but identification amino acids responsible for actin interaction needs further investigation.

\section{Full length nPIST unable to bundle actin filaments:}

nPIST having multiple actin binding motifs, we hypothesized that it might act as a filamentous actin bundling protein. To study that, we incubated $1 \mu \mathrm{M}, 2 \mu \mathrm{M}$ and $3 \mu \mathrm{M}$ of nPIST with $5 \mu \mathrm{M}$ actin and the reactions are centrifuged at much lower speed; 10,000 rpm (Shimada et al.; 2004). Human Fascin1 was used as a positive control. The supernatant and pellet fractions of each reaction were run in SDS PAGE. It displayed that most of the actin when incubated with nPIST was present in supernatant fraction like that of actin control reaction whereas actin majorly appeared in the pellet fraction in presence of positive control Fascin (Maekawa et al.; 1982). nPIST was also mainly present in supernatant fraction along with the actin (Fig. S1A). It indicated that full length nPIST did not bundle actin filaments.

\section{nPIST acts as actin stabilizing agent:}

We further analyzed the effect of full length nPIST on in vitro actin dynamics. In dilution dependent depolymerization assay, 50\% pyrene-labeled preassembled F-actin filaments diluted to $0.1 \mu \mathrm{M}$ concentration were used. The pyrene fluorescence gradually decreased with time which denoted depolymerization of F-actin filaments (Fig 6A and S1B). In presence of nPIST, prevention of depolymerization of F-actin filament is indicated by reduction in fluorescence decrease rate (Fig 6A and B). Xenopus cofilin1 (Xac1) was used as negative control (Rosenblatt et al.; 1997) and when incubated with F-actin, it severed the F-actin filaments which can be visualized by rapid drop in fluorescence intensity. When nPIST was provided in the reaction, it inhibited the F-actin (diluted to $0.25 \mu \mathrm{M}$ ) severing effect of Xac1 in a concentration dependent manner (Fig S1B). We also tested the effect of nPIST on depolymerisation of F-actin filaments diluted to $0.25 \mu \mathrm{M}$ but there was not much change from actin control reaction (Fig. S1C). This notified that full length nPIST stabilized F-actin filaments in vitro and inhibited Xac1 mediated F-actin severing.

\section{nPIST causes unusual accumulation of cellular actin through CC-WH2 domain:}


Next, we looked into the effect of nPIST and its fragments on in vivo actin cytoskeleton. Nterminal GFP-tagged full length nPIST, CC-WH2 $\left(1^{\text {st }}-267^{\text {th }}\right.$ aa $)$, and $\Delta \mathrm{WH} 2\left(268^{\text {th }}-463^{\text {rd }}\right.$ aa $)$ fragment were transiently transfected in cultured HEK293 cells (Fig. 7). Cells were fixed 36 hours post transfection and actin was visualized by rhodamine phalloidin staining ( $1^{\text {st }}$ column). GFP and rhodamine channel merged ( $3^{\text {rd }}$ column) images showed that nPIST was primarily localized in the whole cytoplasm as well as got confined near nucleus to a great extent ( $3^{\text {rd }}$ row). The expression of CC-WH2 fragment ( $4^{\text {th }}$ row) was almost exclusively restricted to perinuclear region of the cell but $\Delta \mathrm{WH} 2$ fragment which did not have golgi localization region $\left(5^{\text {th }}\right.$ row $)$ lost perinuclear expression pattern. Interestingly, nPIST and CC-WH2 fragment co-localized with a patch of actin. On the other hand $\Delta \mathrm{WH} 2$ fragment did not show such effect on actin cytoskeleton which was similar to that of only GFP expression $\left(2^{\text {nd }}\right.$ row). From the above experiment we could suggest that transient over expression of nPIST causes abnormal clustering of F-actin inside cultured HEK293 cells.

\section{Discussion:}

In the current study, we have depicted nPIST as a novel actin binding protein. In silico model of nPIST WH2 domain $\left(233^{\text {th }}-249^{\text {th }}\right.$ aa) and G-actin complex implemented that nPIST may bind to actin adequately (Fig. 1E). Our experimental data validated the in silico data and for the first time we report nPIST acting as an actin binding protein (Fig 2C). Further sequence based analysis of the protein disclosed that nPIST contains several other motifs which act as actin binding site for the protein. We indicated the presence of another three actin binding regions in coiled-coil helix 1 region $\left(\mathrm{CC} 1,1^{\text {st }}-138^{\text {th }}\right.$ aa), coiled-coil helix 2 region $\left(140^{\text {th }}-210^{\text {th }}\right.$ aa) and C-terminal PDZ domain region $\left(\Delta \mathrm{WH} 2,268^{\text {th }}-463^{\text {rd }}\right.$ aa) (Fig. 4A, and $\left.5 \mathrm{~A}\right)$. F-actin cosedimentation assay results showed that each of these fragmented regions can individually bind to actin (Fig. 4D, 5F, 5G, and 5H). However, to determine the exact amino acids residues involved in actin binding needs further detailed study. The presence of several actin binding regions makes nPIST capable of binding to actin. Our experimental data also showed that nPIST can stabilize F-actin filaments in vitro and inhibited filament severing effect of Xac1 (Fig. 6A, 6B, S1B and S1C). We have also investigated the effect of nPIST on actin cytoskeleton in vivo (Fig. 7). Full length nPIST and $\Delta \mathrm{WH} 2\left(268^{\text {th }}-463^{\text {rd }}\right.$ aa) was found to be expressing throughout the cell cytosol (Fig. $7,3^{\text {rd }}$ and $5^{\text {th }}$ row) whereas CC-WH2 $\left(1^{\text {st }}-267^{\text {th }}\right.$ aa) was exclusively 
localized to a perinuclear region of cytoplasm (Fig. 7, $4^{\text {th }}$ row). nPIST and CC-WH2 fragment co-localized with phalloidin stained actin patch but such phenomenon was not observed in case of $\Delta \mathrm{WH} 2$ fragment. Occurance of similar kind of F-actin accumulation can also be seen in case of overexpression of Slingshot phosphatase (Soosairajah et al., 2005) but not in juxtanuclear region. The mechanism behind the occurrence of such actin patch needs further inspection to unfold the underlying story. Strikingly, nPIST and the CC-WH2 fragment consisting of golgi localization region in the second coiled-coil helix region got spatially localized to the juxtanuclear region. On the other hand, the $\Delta \mathrm{WH} 2$ fragment lacking golgi localizing region lost its targeted expression and got distributed in the whole cytosol. Whether the co-localization of nPIST and actin was in golgi region or not can be disclosed by co-localization study with golgi resident markers. The actin binding regions were mostly present within CC-WH2 fragment and nPIST was capable of co-localizing actin through its CC-WH2 fragment but $\triangle \mathrm{WH} 2$ fragment having only one of such stretch interacting with actin domain did not exert much any effect on cellular actin cytoskeleton. Till date studies have suggested that WASP family proteins have evolved to mostly promote Arp2/3 complex mediated actin nucleation (Kollmar et al.; 2012). But some members of this family have Arp2/3 independent activity and directly function as actin nucleating candidate (Dominguez et al.; 2016). Our candidate protein nPIST is another WH2like domain containing protein that directly interacts with actin and modulates its dynamics in vitro.

The proper positioning and maintenance of golgi morphology requires presence of actin microfilaments (Valderrama et al.; 1998). Several actin binding proteins located in golgi network have been reported to be linking actin cytoskeleton to vesicular trafficking regulation, modeling of golgi architecture and integrating molecular machinery for vesicular transport (Egea et al.; 2006). Among these microfilament regulators, there are several Wiskott-Aldrich syndrome protein (WASP) family candidates involved in different compartments of vesicle mediated transport pathway. WHAMM is known to regulate trafficking in ER-Golgi intermediate compartment (ERGIC) compartments (Kenneth et al.; 2008). The closest family member of WHAMM; JMY on the other hand is mainly involved in the trans-Golgi network (TGN) anterograde trafficking (Schlüter et al.; 2014). Another WASP family member WASH functions in regulation of endosome shape and fission (Derivery et al.; 2009, Duleh and Welch; 2010). The molecular mechanism underlying nPIST mediated vesicular trafficking regulation in terms 
of actin cytoskeleton regulation remains to be elucidated which can be an important functional aspect to be explored that can have considerable contribution in the broad spectrum of purpose served by WASP family proteins (Alekhina et al.; 2017).

\section{Materials and methods:}

Computational Analysis of nPIST WH2 domain:

The structural model of nPIST was obtained using Swiss model and modeller (Kelley et al.; 2009). Sequences of WH2 domain from all the crystal structure available were extracted and aligned with the sequence of nPIST, resulted in identification of $\mathrm{WH} 2$ domain in the region $\left(233^{\text {rd }}-249^{\text {th }}\right.$ aa) of nPIST. The model of G-actin-WH2 domain complex was obtained using the cross-linked complex of actin with first WH2 domain of $V$. parahaemolyticus VopL (PDB ID: 3M1F) (Reboeski et al.; 2010). The WH2-domain was docked using rosetta Flexpepdock server (London et al.; 2011). The starting structure was refined in 200 independent FlexPepDock simulations. 100 of simulations were carried out strictly in high-resolution mode, while 100 of simulation included a low-resolution pre-optimization step, followed by high-resolution refinement. A total of 200 models were created and then ranked based on their Rosetta generic full-atom energy score. The final model of the complex was further energy minimized using AMBER12 molecular dynamics software (Case et al.; 2012). Analysis of the complex using KFC-2 server (Zhu et al.; 2011), predicted binding "hot spots" within protein-protein interfaces by recognizing structural features indicative of important binding contacts. The figures were prepared using Pymol software (The PyMOL Molecular Graphics System, Version 1.5.0.4 Schrödinger, LLC).

Plasmid constructs:

Total RNA was isolated using TRIzol (Invitrogen) from adult brain tissue of C57BL/6 mouse; cDNA was prepared by RT-PCR (Superscript III, Invitrogen), and full length sequence was amplified with forward 5'GGAAGATCTATGTCGGCGGGTGGC3' and reverse 5'CCGCGTCGACTTAGTAGGCCTTCTTCTGATGCA3' primers. Full length nPIST was cloned in pET-28a vector (Novagen) using BamHI and SalI, expressed in E. coli BL21-DE3-RP as N-terminal 6-His tagged protein. The sub clones of nPIST fragments were prepared in similar manner. The primer used for sub-cloning were; forward 
5'GGAAGATCTATGTCGGCGGGTGGC3'

and

reverse

$5^{\prime}$

CCGCGTCGACTTACATGGGCCGCTTCAAG $3^{\prime}$ for CC-WH2 $\left(1^{\text {st }}-267^{\text {th }}\right.$ aa) fragment, forward 5'GGAAGATCTCAAGCACCCCCAGGCC3'

and

reverse

5'CCGCGTCGACTTAGTAGGCCTTCTTCTGATGCA3'for $\Delta \mathrm{WH} 2 \quad\left(268^{\text {th }}-463^{\text {rd }}\right.$ aa) fragment, forward 5'GGAAGATCTATGTCGGCGGGTGGC3' and reverse 5'CCGCGTCGACTTAAGTCTTGGCATGAAGCTGAAG3' for CC1 ( $1^{\text {st }}-138^{\text {th }}$ aa) fragment, forward 5'GGAAGATCTCAAAGTGTTGACTCTGGGG3' and reverse 5'CCGCGTCGACTTAGTACTTGGCAGCCAACCTC3' for CC2 $\left(140^{\text {th }}-210^{\text {th }}\right.$ aa $)$ fragment, forward 5'GGAAGATCTGAAGTTTATGGGGCGAGG3' and reverse 5' CCGCGTCGACTTATTTTCTAATTGGACCGACTC3' for WH2 $\left(200^{\text {th }}-289^{\text {th }}\right.$ aa $)$ fragment, and forward 5' GGAAGATCTCCAATTAGAAAAGTTCTCCTCC 3' and reverse 5' CCGCGTCGACTTAGTAGGCCTTCTTCTGATGCA3' for PDZ $\left(285^{\text {th }}-463^{\text {rd }}\right.$ aa) fragment. Human Fascin1 construct was a kind gift from Professor Jo Adams and Dr. Aurnab Ghose. It was sub-cloned in pET28a vector using forward 5, GACGAGGATCCATGACCGCCAACGGCACAG3' ${ }^{\prime}$ and CAGCTCGAGCTAGTACTCCCAGAGCGAGGCG3’ primers.

Protein Purification:

G-actin was purified from rabbit muscle acetone powder (Pollard TD; 1984) with G-Buffer [5 mM Tris pH 8.0 (Sigma-Aldrich), $0.2 \mathrm{mM}$ ATP (USB), $0.2 \mathrm{mM} \mathrm{CaCl}_{2}$ (USB) and $0.2 \mathrm{mM}$ DTT (USB)]. Actin was taaged with N-(-1-pyrene) iodoacetamide (P-29, Molecular probe) for fluorescence spectroscopic analysis (Higgs and Pollard, 1999). E. coli BL21-DE3-RP (Stratagene) containing nPIST, its different fragment constructs and Fascin1 were grown up to O.D 0.6 at $37^{\circ} \mathrm{C}$, induced with $0.5 \mathrm{mM}$ IPTG (Thermo Fisher Scientific), harvested, and stored in $-80^{\circ} \mathrm{C}$ (Moseley et al.; 2006). Harvested cells were resuspended in lysis buffer [50 mM Tris-Cl pH 8.0, 100 mM NaCl (Sigma-Aldrich), 30 mM Imidazole pH 8.0 (USB), 0.5 mM DTT, 0.5\% IGEPAL (Sigma-Aldrich), and Protease Inhibitor Cocktail], lysed by sonication for 5 minutes in 1 minute pulse in 30 second interval, and then centrifuged at $12000 \mathrm{rpm}$. The supernatant was incubated with $50 \%$ slurry of Ni-NTA resin beads (Qiagen), washed with wash buffer (50 mM Tris-Cl pH8.0, $150 \mathrm{mM} \mathrm{NaCl}, 50 \mathrm{mM}$ Imidazole pH8.0) and finally eluted with elution buffer [50 mM Tris-Cl pH8.0, $100 \mathrm{mM} \mathrm{NaCl}, 350 \mathrm{mM}$ Imidazole $\mathrm{pH} 8.0$, and 5\% glycerol 
(Invitrogen)]. Next, purified cloned constructs were dialyzed in $\mathrm{HEKG}_{5}$ buffer [20 mM HEPES (USB), 1 mM EDTA (Calbiochem), 50 mM KCl (Sigma-Aldrich), and 5\% Glycerol) (Moseley et al.; 2006). nPIST and its fragments were further purified using Superdex-200 10/300 GL column (GE Healthcare).

Xenopus cofilin 1 in pGEX_(GST-Xac1) was kindly gifted by Prefessor JR Bamburg (Abe et al.; 1996). GST-tagged Xac1 was expressed in E. coli BL21-DE3 (Stratagene) at 37oC and induced with $0.5 \mathrm{mM}$ IPTG for 4 hours. Post harvestion, cells were resuspended in lysis buffer (50 mM Tris-Cl pH 8.0, $150 \mathrm{mM} \mathrm{NaCl}, 1 \mathrm{mM}$ DTT, $1 \mathrm{mM}$ DTT, 0.5\% IGEPAL, and Protease Inhibitor Cocktail), sonicated, incubated with $50 \%$ slurry of GST-Agarose bead (thermo Scientific) and eluted with elution buffer having $50 \mathrm{mM}$ Tris- $\mathrm{Cl} \mathrm{pH}$ 8.0, $150 \mathrm{mM} \mathrm{NaCl}, 1 \mathrm{mM}$ EDTA, and 10 mM Reduced Glutathione (USB).

Co-sedimentation Assay:

$5 \mu \mathrm{M}$ G-actin was polymerized into F-actin for 1 hour at $25^{\circ} \mathrm{C}$ in F-buffer $[10 \mathrm{mM}$ Tris-Cl pH 8.0, $0.2 \mathrm{mM}$ DTT, $0.5 \mathrm{mM}$ ATP, $50 \mathrm{mM} \mathrm{KCl}$, and $2 \mathrm{mM} \mathrm{MgCl}_{2}$ (Sigma-Aldrich), $0.2 \mathrm{mM}$ $\mathrm{CaCl}_{2}$ ]; subsequently nPIST was added for 15 minutes and then centrifuged at $310 \mathrm{x} 1000 \mathrm{~g}$ for 40 minutes in TLA-100 rotor (Beckman Coulter). Supernatant was separated from pellet fraction and pellet was resuspended in F-buffer. All the samples were boiled with $4 \mathrm{X}$ sample loading buffer, loaded on SDS-PAGE, visualized with coomassie (SRL) staining. The above procedure was carried out for different fragments of nPIST to check their actin binding property (Shimada et al.; 2004).

Interaction kinetics by Surface Plasmon Resonance (SPR):

SPR experiments were performed using a Biacore T200 system. All experiments were conducted in duplicates at $25^{\circ} \mathrm{C}$ and double referencing (blank immobilized channel and blank injections) was used to eliminate bulk effects. nPIST (ligand) was immobilized on a series S CM5 sensor chip using amine coupling to achieve an immobilization level of $\sim 1500$ RUs. The running buffer was varied according to the analyte being used (G-buffer for G-actin and F-buffer for F-actin). Varying concentrations of the analytes $(0.5 \mu \mathrm{M}, 1 \mu \mathrm{M}, 2 \mu \mathrm{M}, 4 \mu \mathrm{M}$, and $8 \mu \mathrm{M})$ were passed over the immobilized ligand at a flow rate of $10 \mu \mathrm{l} / \mathrm{min}$ for $180 \mathrm{~s}$ to observe the association phase 
and the dissociation was observed by flowing blank buffer for $120 \mathrm{~s}$. Data analyses were carried out with Biacore T200 evaluation software 2.0 to fit the data to 1:1 interaction model.

F-actin depolymerization assay:

$5 \mu \mathrm{M}$ of $50 \%$ pyrene-labeled rabbit muscle actin was incubated in F-buffer and polymerized into F-actin for one hour at $25^{\circ} \mathrm{C}$. From this preassembled actin, $2 \mu 1$ was used in $100 \mu$ l of reaction having $83 \mu \mathrm{l}$ of F-buffer and $15 \mu \mathrm{l}$ of HEKG5 buffer or protein samples. The N-pyrene (Invitrogen) fluorescence was excited at $365 \mathrm{~nm}$ and the emitted spectrum was measured at 407 nm for 600 seconds (QM 40 PTI NJ) (Rosenblatt et al.; 1997).

Cellular Experimentation:

HEK 293 cells (ATCC® -CRL-1573) were grown in poly D-lysine coated cover slips in 24-well plate with MEM (Gibco) containing L-Glutamine (Gibco), penicillin streptomycin (Hyclone, Thermo Scientific) and 10\% Fetal Bovine Serum (Gibco). Full length nPIST and its CC-WH2 $\left(1^{\text {st }}-267^{\text {th }}\right.$ aa) and $\Delta \mathrm{WH} 2\left(268^{\text {th }}-463^{\text {rd }}\right.$ aa) fragments were cloned in pEGFPC1 vector (Stratagene). Cells were transfected with cloned nPIST constructs with Lipofectamine 2000 (Invitrogen) following manufacturer's protocol. The cells were fixed using 4\% paraformaldehyde (Sigma-Aldrich), 36 hours post transfection. Cellular actin was visualized with $50 \mathrm{nM}$ rhodamine phalloidin staining (Invitrogen) and nPIST and its fragments were ectopically expressed as N-terminal GFP tagged proteins. The cell containing cover slips were mounted with antifade reagent (Thermo Fisher Scientific) and images were captured with laser scanning microscope (Carl Zeiss LSM710) using 488 laser and 561 laser.

\section{Acknowledgements:}

SD thanks IISER-Kolkata and MM thanks ICMR for providing their fellowship. Xac1-GST was gifted to SM by Prof JR Bamburg. Human Fascin1 cloned in pEGFP-C1 was a kind gift from Professor Jo Adams and Dr. Aurnab Ghose. This work is supported by IISER Kolkata.

\section{Conflict of interest}

Authors declare no conflict of interest in the concerned area of research. 


\section{Authors' contrubutions:}

Designed and conceived the experiment: SM, PD, SD, SS, KD, DS, MM and SG. Experiments performed by: PD, SD, MM, SS, KD. Analyzed the data: SM, PD, SD, SS, KD, DS, MM and SG. Writing of the manuscript: SM, PD, SD, SS, KD, DS, MM and SG.

\section{Figure Legends:}

Figure 1: Structure based modelling of nPIST indicated the presence of putative WH2 domain in nPIST

(A) The template identification carried out by Swiss model is shown in figure. The scematics shows the sequence coverage of closely related models available in PDB database with characterized domains labeled B) The models of N-terminal coiled-coil domain region of nPIST C) Models of C-terminal PDZ domain region nPIST with identified WH2 domain (D) The Structure based sequence alignment of $\mathrm{WH} 2$ domain sequences obtained from crystal structure of various actin-WH2 domain complexes with nPIST. Binding of probable WH2 domain of nPIST with G-Actin; (E) Two $180^{\circ}$ view of the model of G-actin-WH2 Domain complex. (F) The location of hotspots at the binding interface of G-actin predicted by KFC2 server.

\section{Figure 2: Full length nPIST can bind to actin}

(A) Schematic representation of full length nPIST. (B) Purified full length nPIST $\left(1^{\text {st }}-463^{\text {rd }}\right.$ aa) in coomassie stained 12\% SDS-PAGE. The protein is expressed in E. coli B121-DE3-RP cells as N-terminal 6-His tagged and purified using Ni-NTA-agarose bead. (C) SDS-PAGE of supernatant $(\mathrm{S})$ and pellet $(\mathrm{P})$ fraction of actin co-sedimentation assay with $0.5-5 \mu \mathrm{M}$ of full length nPIST.

\section{Figure 3: SPR interaction analysis for F-actin binding to nPIST}

The sensorgram shows association and dissociation phases of varying concentrations of F-actin (analyte) binding to nPIST (immobilized ligand). The interactions follow rapid kinetics. Fitting the data to $1: 1$ interaction model gives a $K_{\mathrm{D}}$ of $\sim 13.3 \mathrm{nM}$ for F-actin.

\section{Figure 4: Fragment of nPIST lacking WH2 domain also binds to actin}


(A) Illustrative portrayal of fragments nPIST showing N-terminal coiled-coil domain containing construct (CC-WH2) and C-terminal PDZ domain containing construct $(\triangle \mathrm{WH} 2)$. (B) SDSPAGE of 6-His tagged purified protein of CC-WH2 (lane 1) and $\triangle \mathrm{WH} 2$ (lane 2) fragments of nPIST. Supernatant (S) and pellet (P) fraction of actin co-sedimentation assay of CC-WH2 (C) and $\triangle \mathrm{WH} 2$ (D) fragments separated by $12 \%$ SDS-PAGE and stained with coomassie.

Figure 5: Presence of several actin interacting regions makes nPIST an actin binding protein

(A) Schematic diagram of truncated fragments of nPIST considered to identify actin binding regions. 6-His tagged purified protein of (B) CC1 fragment $\left(1^{\text {st }}-138^{\text {th }}\right.$ aa), (C) CC2 fragment $\left(140^{\text {th }}-210^{\text {th }}\right.$ aa), (D) WH2 fragment $\left(200^{\text {th }}-289^{\text {th }}\right.$ aa), and (E) PDZ fragment $\left(285^{\text {th }}-463^{\text {rd }}\right.$ aa) of nPIST run in coomassie stained 12\% SDS-PAGE. Gel electrophoresis of supernatant and pellet fraction of F-actin co-sedimentation assay reactions of (F) CC1 fragment, (G) CC2 fragment, $(\mathrm{H})$ WH2 fragment, and (I) PDZ fragment of nPIST.

Figure 6: nPIST stabilizes F-actin filaments in vitro

(A) F-actin filament stabilization assay with $0.1 \mu \mathrm{M} 50 \%$ pyrene-labeled actin in presence of increasing concentration of nPIST. Хеnориs cofilin 1 (Xac1) used as filament stabilization negative control and severed actin filaments marked by the rapid decrease in fluorescence intensity of N-pyrene. (B) Actin filament stabilization rate measured as the slope of fluorescence curve for 10-100 secs.

\section{Figure 7: Effect of Full Length nPIST and its Fragments on Cellular Actin Cytoskeleton}

Full length nPIST and its fragments (CC-WH2 and $\triangle \mathrm{WH} 2)$ was sub cloned in pEGFP-C1 vector. HEK 293 cells were transfected with GFP vector control $\left(2^{\text {nd }}\right.$ row), full length nPIST ( $3^{\text {rd }}$ row), $\mathrm{CC}-\mathrm{WH} 2$ fragment ( $4^{\text {th }}$ row), and $\Delta \mathrm{WH} 2$ fragment $\left(5^{\text {th }}\right.$ row). Actin was stained with rhodaminephalloidin $\left(1^{\text {st }}\right.$ column $)$. Third column shows merged actin and GFP.

\section{Figure S1: Full length nPIST does not bundle but stabilizes actin filaments}

(A) In vitro low speed F-actin co-sedimentation assay of full length nPIST performed to check its bundling ability. $5 \mu \mathrm{M}$ actin and 1,2 and $3 \mu \mathrm{M}$ of nPIST incubation reaction was centrifuged 
at $9.2 \times 1000 \mathrm{~g}$ for 10 minutes at $4^{\circ} \mathrm{C}$ and supernatant and pellet fraction run in SDS-PAGE is denoted by $\mathrm{S}$ and $\mathrm{P}$ respectively. $3 \mu \mathrm{M}$ human Fascin1 was used as positive control. (C) Actin filament stabilization assay with $0.25 \mu \mathrm{M}$ of $50 \%$ pyrene-labeled actin. nPIST salvaged the actin filaments from being severed by negative control Xac1 in dose dependent manner. (D) Dilution dependent depolymerization assay of only $0.25,0.5$ and $1 \mu \mathrm{M}$ of nPIST with $0.25 \mu \mathrm{M}$ of actin where Xac1 was used as negative control. (E) SPR sensorgram for the binding of G-actin to immobilized nPIST, showing the instable interaction.

\section{References:}

1. Abe H, Obinata T, Minamide LS, Bamburg JR. (1996). Xenopus laevis actindepolymerizing factor/cofilin: a phosphorylation-regulated protein essential for development. Journal Cell Biology 132(5):871-85.

2. Alekhina O, Burstein E, Billadeau DD. (2017). Cellular functions of WASP family proteins at a glance. J Cell Science 15; 130(14):2235-2241.

3. Campellone KG, Webb NJ, Znameroski EA, Welch MD (2008). WHAMM Is an Arp2/3 Complex Activator That Binds Microtubules and Functions in ER to Golgi Transport. Cell 134 (1), 148-61.

4. Case DA, Darden TA, Cheatham TE, Simmerling CL, Wang J, Duke RE, Luo R, Walker RC, Zhang W, Merz KM, Roberts B, Hayik S, Roitberg A, Seabra G, Swails J, Goetz AW, Kolossváry I, Wong KF, Paesani F, Vanicek J, Wolf RM, Liu J, Wu X, Brozell SR, Steinbrecher T, Gohlke H, Cai Q, Ye X, Wang J, Hsieh MJ, Cui G, Roe DR, Mathews DH, Seetin MG, Salomon-Ferrer R, Sagui C, Babin V, Luchko T, Gusarov S, Kovalenko A, and Kollman PA (2012). AMBER 12. University of California, San Francisco.

5. Charest A, Lane K, McMahon K, Housman DE (2001). Association of a novel PDZ domain-containing peripheral Golgi protein with the Q-SNARE (Q-soluble Nethylmaleimide-sensitive fusion protein (NSF) attachment protein receptor) protein syntaxin 6. Journal Biological Chemistry 276(31), 29456-65. 
6. Charest A, Lane K, McMahon K, Park J, Preisinger E, Conroy H, Housman D (2003). Fusion of FIG to the receptor tyrosine kinase ROS in a glioblastoma with an interstitial del(6)(q21q21). Genes Chromosomes Cancer 37(1), 58-71.

7. Chen A, Gößling EK, Witkowski L, Bhindi A, Bauch C, Roussy G, Sarret P, Kreienkamp HJ, and Stroh T (2012). Regional and Subcellular Distribution of the Receptor-Targeting Protein PIST in the Rat Central Nervous System. The Journal of Comparative Neurology 520, 889-913.

8. Cheng J, Moyer BD, Milewski M, Loffing J, Ikeda M, Mickle JE, Cutting GR, Li M, Stanton BA, Guggino WB (2002). A Golgi-associated PDZ domain protein modulates cystic fibrosis transmembrane regulator plasma membrane expression. Journal Biological Chemistry 277(5), 3520-9.

9. Cheng J, Wang H, Guggino WB (2004). Modulation of mature cystic fibrosis transmembrane regulator protein by the PDZ domain protein CAL. Journal Biological Chemistry 279(3), 1892-8.

10. Cheng J, Cebotaru V, Cebotaru L, Guggino WB (2010). Syntaxin 6 and CAL mediate the degradation of the cystic fibrosis transmembrane conductance regulator. Molecular Biology of the Cell 21(7):1178-87.

11. Cheng J, Guggino W (2013). Ubiquitination and degradation of CFTR by the E3 ubiquitin ligase MARCH2 through its association with adaptor proteins CAL and STX6. PLoS One 8(6), e68001.

12. Cheng S, Zhang J, Zhu P, Ma Y, Xiong Y, Sun L, Xu J, Zhang H, He J (2010). The PDZ domain protein CAL interacts with mGluR5a and modulates receptor expression. Journal Neurochemistry 112(3), 588-98.

13. Cuadra AE, Kuo SH, Kawasaki Y, Bredt DS, Chetkovich DM (2004). AMPA receptor synaptic targeting regulated by stargazin interactions with the Golgi-resident PDZ protein nPIST. Journal of Neuroscience 24, 7491-7502. 
14. Derivery E, Sousa C, Gautier JJ, Lombard B, Loew D, and Gautreau A (2009). The Arp2/3 Activator WASH Controls the Fission of Endosomes through a Large Multiprotein Complex. Developmental Cell 17 (5), 712-23.

15. Dominguez R, Holmes KC (2011). Actin structure and function. Annual Review of Biophysics 40, 169-86.

16. Dominguez R. (2016). The WH2 Domain and Actin Nucleation: Necessary but Insufficient. Trends Biochem Sci. 41(6):478-490.

17. Duleh SN, and Welch MD (2010). WASH and the Arp2/3 complex regulate endosome shape and trafficking. Cytoskeleton 67 (3), 193-206.

18. Egea G, Die'guez FL, and Vilella M (2006). Actin dynamics at the Golgi complex in mammalian cells. Current Opinion in Cell Biology 18, 168-78.

19. Gentzsch M, Cui L, Mengos A, Chang XB, Chen JH, Riordan JR (2003). The PDZbinding chloride channel $\mathrm{ClC}-3 \mathrm{~B}$ localizes to the Golgi and associates with cystic fibrosis transmembrane conductance regulator-interacting PDZ proteins. Journal Biological Chemistry 278(8), 6440-9.

20. Hammad MM, Dunn HA, Walther C, Ferguson SS (2015). Role of cystic fibrosis transmembrane conductance regulator-associated ligand $(\mathrm{CAL})$ in regulating the trafficking and signaling of corticotropin-releasing factor receptor 1. Cellular Signalling 27(10), 2120-30.

21. Hicks SW, Machamer CE (2005). Isoform-specific interaction of golgin-160 with the Golgi-associated protein PIST. Journal Biological Chemistry 280(32), 28944-51.

22. Higgs HN and Pollard TD (1999). Regulation of Actin polymerization by Arp2/3 Complex and WASp/Scar Proteins. The Journal of Biological Chemistry, 274(46), $32531-34$. 
23. He J, Bellini M, Xu J, Castleberry AM, Hall RA (2004). Interaction with cystic fibrosis transmembrane conductance regulator-associated ligand (CAL) inhibits beta1-adrenergic receptor surface expression. Journal Biological Chemistry 279(48), 50190-6.

24. Ito C, Suzuki-Toyota F, Maekawa M, Toyama Y, Yao R, Noda T, Toshimori K (2004). Failure to assemble the peri-nuclear structures in GOPC deficient spermatids as found in round-headed spermatozoa. Archives of Histology and Cytology 67(4), 349-60.

25. Ito H, Iwamoto I, Morishita R, Nozawa Y, Asano T, Nagata K (2006). Identification of a PDZ protein, PIST, as a binding partner for Rho effector Rhotekin: biochemical and cellbiological characterization of Rhotekin-PIST interaction. Biochemical Journal 397(3), 389-98.

26. Kelley, LA, and Sternberg, MJ (2009). Protein structure prediction on the Web: a case study using the Phyre server. Nature protocols 4, 363-371.

27. Kollmar, M., Lbik, D. and Enge, S. (2012). Evolution of the eukaryotic ARP2/3 activators of the WASP family: WASP, WAVE, WASH, and WHAMM, and the proposed new family members WAWH and WAML. BMC Research Notes 5, 88.

28. Li M, Soroka CJ, Harry K, Boyer JL (2017). CFTR-associated ligand is a negative regulator of Mrp2 expression. Americal Journal of Physiology Cell Physiology 312(1), C40-C46.

29. London N, Raveh B, Cohen E, Fathi G, and Schueler-Furman O (2011). Rosetta FlexPepDock web server--high resolution modeling of peptide-protein interactions. Nucleic acids research 39, W249-253.

30. Lu R, Stewart L, Wilson JM (2015). Scaffolding protein GOPC regulates tight junction structure. Cell and Tissue Research 360(2), 321-32.

31. Maekawa S, Endo S, Sakai H (1982). A protein in starfish sperm head which bundles actin filaments in vitro: purification and characterization. The Journal of Biochemistry 92(6), 1959-72. 
32. Moseley JB, Maiti S, Goode BL (2006). Formin proteins: purification and measurement of effects on actin assembly. Methods Enzymol. 406, 215-34.

33. Neudauer CL, Joberty G, Macara IG (2001). PIST: A Novel PDZ/Coiled-Coil Domain Binding Partner for the Rho-Family GTPase TC10. Biochemical and Biophysical Research Communications 280, 541-547.

34. Nie H, Liu Y, Yin X, Cao H, Wang Y, Xiong W, Lin Y, Xu Z (2016). Plasma Membrane Targeting of Protocadherin 15 Is Regulated by the Golgi-Associated Chaperone Protein PIST. Neural Plasticity 2016, 8580675.

35. Paunola E, Mattila PK, Lappalainen P (2002). WH2 domain: a small, versatile adapter for actin monomers. FEBS Letters 513, 92-97.

36. Pollard TD (1984). Polymerization of ADP-actin. Journal of Cell Biology 99, 769-77.

37. Rebowski, G, Namgoong, S, Boczkowska, M, Leavis, PC, Navaza, J, and Dominguez, R (2010). Structure of a longitudinal actin dimer assembled by tandem $\mathrm{W}$ domains: implications for actin filament nucleation. Journal of molecular biology 403, 11-23.

38. Rosenblatt J, Agnew BJ, Abe H, Bamburg JR, Mitchison TJ (1997). Xenopus actin depolymerizing factor/cofilin (XAC) is responsible for the turnover of actin filaments in Listeria monocytogenes Tails. The Journal of Cell Biology 136 (6), 1323-1332.

39. Schlüter K, Waschbüsch D, Anft M, Hügging D, Kind S, Hänisch J, Lakisic G, Gautreau A, Barnekow A, Stradal TE (2014). JMY is involved in anterograde vesicle trafficking from the trans-Golgi network. European Journal of Cell Biology 93(5-6), 194-204

40. Shimada A, Nyitrai M, Vetter IR, Kühlmann D, Bugyi B, Narumiya S, Geeves MA, and Wittinghofer A (2004). The core FH2 domain of diaphanous-related formins is an elongated actin binding protein that inhibits polymerization. Mol. Cell. 13, 511-22.

41. Shin YC, Seo EK, Jeon JH, Park HH (2013). Crystallization and preliminary X-ray crystallographic studies of the coiled-coil domain of PIST. Acta Crystallogr Sect F Struct Biol Cryst Commun. 69(Pt 4), 468-71. 
42. Soosairajah J, Maiti S, Wiggan O, Sarmiere P, Moussi N, Sarcevic B, Sampath R, Bamburg JR, Bernard O (2005). Interplay between components of a novel LIM kinaseslingshot phosphatase complex regulates cofilin. EMBO Journal 24(3), 473-86.

43. The PyMOL Molecular Graphics System, Version 1.5.0.4 Schrödinger, LLC.

44. Valderrama F, Babia T, Ayala I, Kok JW,Piqueras JR, Egea G (1998). Actin microfilaments are essential for the cytological positioning and morphology of the Golgi complex. European Journal of Cell Biology 76, 9-17.

45. Wente W1, Stroh T, Beaudet A, Richter D, Kreienkamp HJ (2005). Interactions with PDZ domain proteins PIST/GOPC and PDZK1 regulate intracellular sorting of the somatostatin receptor subtype 5. Journal Biological Chemistry 280(37), 32419-25.

46. Yue Z, Horton A, Bravin M, DeJager PL, Selimi F, Heintz N (2002). A Novel Protein Complex Linking the $\delta 2$ Glutamate Receptor and Autophagy: Implications for Neurodegeneration in Lurcher Mice. Neuron 35, 921-933.

47. Yang L, Zheng J, Xiong Y, Meng R, Ma Q, Liu H, Shen H, Zheng S, Wang S, He J (2015). Regulation of $\beta 2$-adrenergic receptor cell surface expression by interaction with cystic fibrosis transmembrane conductance regulator-associated ligand (CAL). Amino Acids 47(7), 1455-64.

48. Yao R, Ito C, Natsume Y, Sugitani Y, Yamanaka H, Kuretake S, Yanagida K, Sato A, Toshimori K, Noda T (2002). Lack of acrosome formation in mice lacking a Golgi protein, GOPC. Proceedings of the National Academy of Sciences of the United States of America 99(17), 11211-6.

49. Yao R, Maeda T, Takada S, Noda T (2001). Identification of a PDZ Domain Containing Golgi Protein, GOPC, as an Interaction Partner of Frizzled. Biochemical and Biophysical Research Communications 286, 771-778.

50. Xu Z, Oshima K, Heller S (2010). PIST regulates the intracellular trafficking and plasma membrane expression of cadherin 23. BMC Cell Biology 11, 80. 
51. Zhang J, Cheng S, Xiong Y, Ma Y, Luo D, Jeromin A, Zhang H, He J (2008). A novel association of mGluR1a with the PDZ scaffold protein CAL modulates receptor activity. FEBS Letters 582(30), 4117-24.

52. Zhu X, and Mitchell JC (2011). KFC2: A knowledge-based hot spot prediction method based on interface solvation, atomic density, and plasticity features. Proteins: Structure, Function, and Bioinformatics 79, 2671-2683. 
Figure 1:

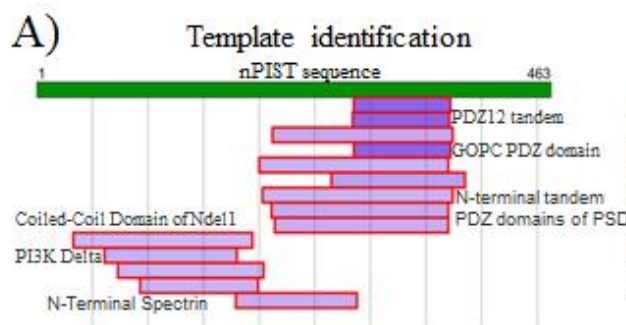

D)

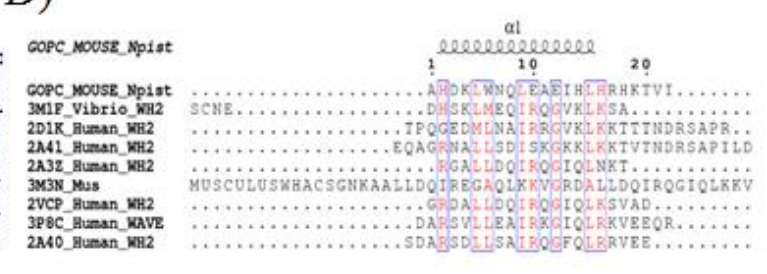

B)

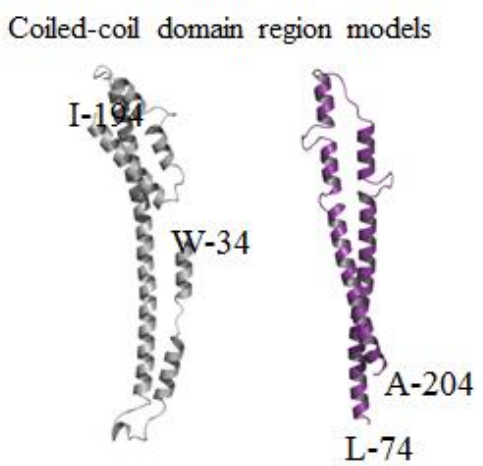

E)

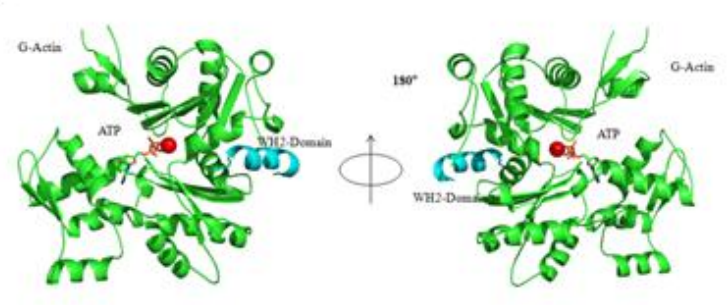

C)

PDZ domain region models

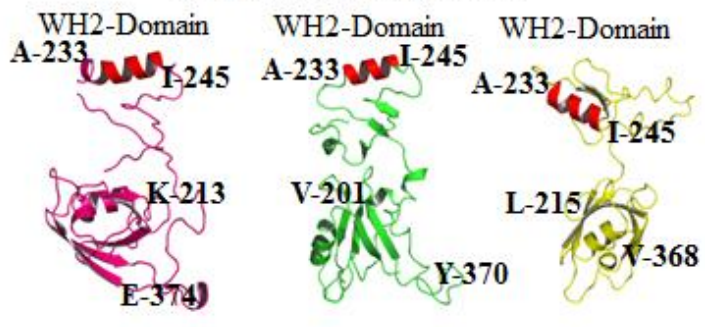

F)

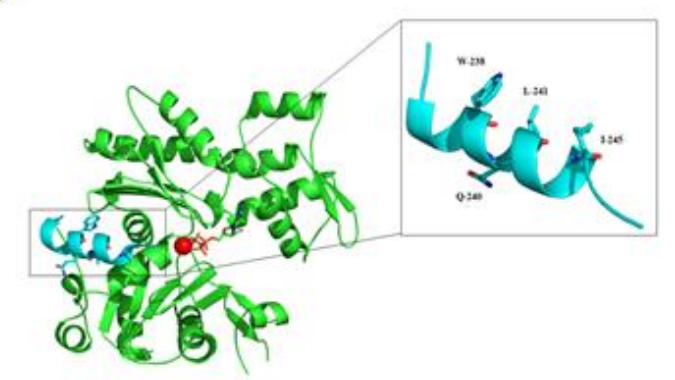

Figure 2:

(A)

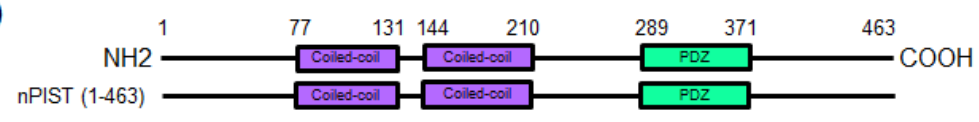

(B)

(C)
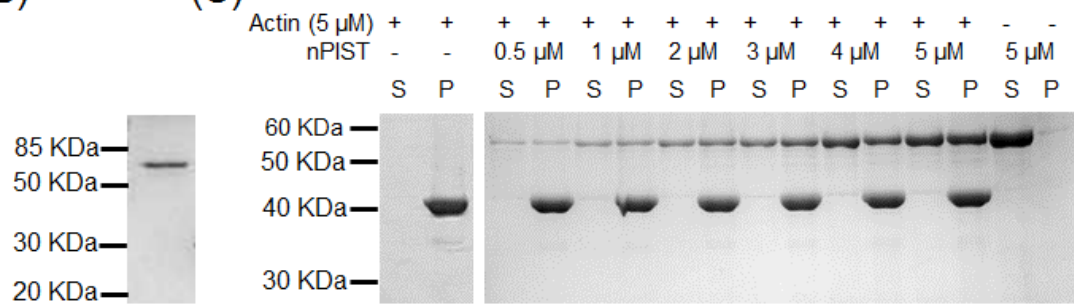
Figure 3:

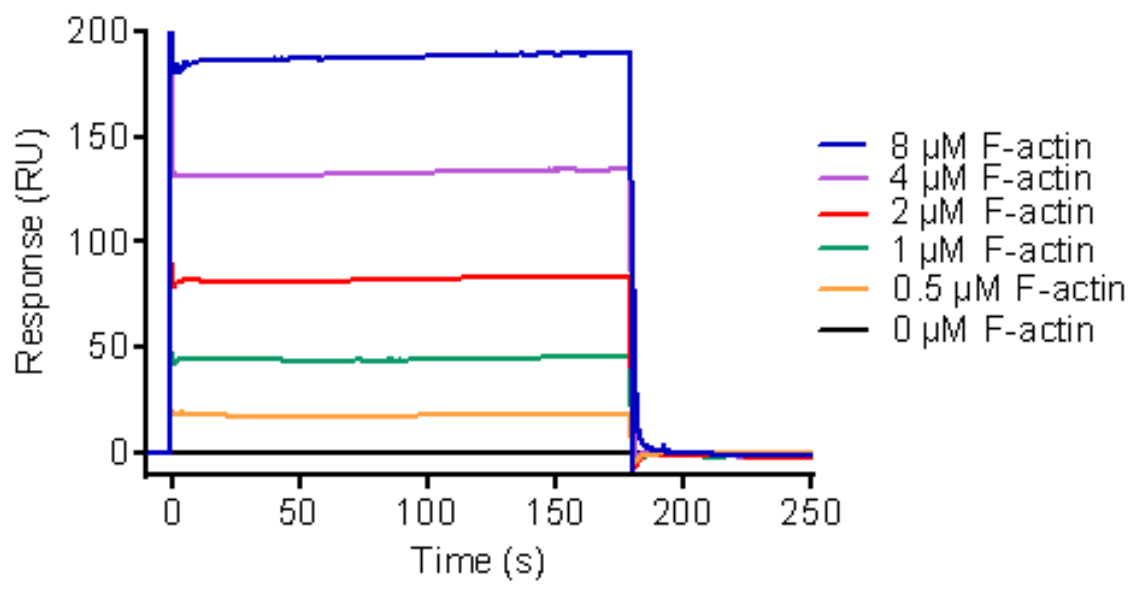

Figure 4:

(A)

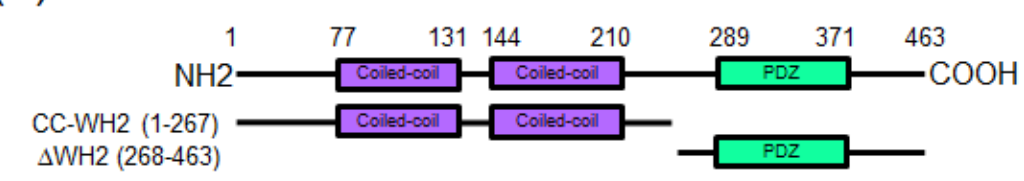

(B)

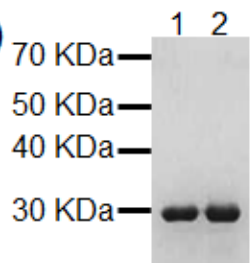

(C) $\operatorname{Actin}(5 \mu \mathrm{M})++++++++\cdots$ nPIST (CC-WH2) - - $4 \mu \mathrm{M} 8 \mu \mathrm{M} 12 \mu \mathrm{M} 12 \mu \mathrm{M}$ $S$ P S P S P S P S P $50 \mathrm{KDa}-$ $30 \mathrm{KDa}-$
(D) $\operatorname{Actin}(5 \mu \mathrm{M})++++++++-$ nPIST $(\Delta \mathrm{WH} 2)$ - $-6 \mu \mathrm{M} 12 \mu \mathrm{M} 18 \mu \mathrm{M} 18 \mu \mathrm{M}$ S P S P S P S P S P $50 \mathrm{KDa}-$ $30 \mathrm{KDa}-$ 
Figure 5:

(A)

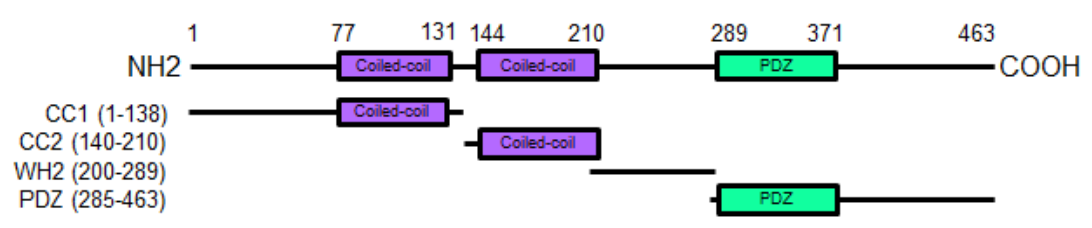

(B)

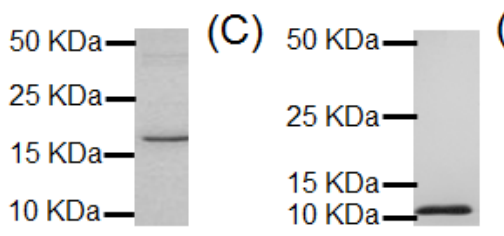

(D) $70 \mathrm{KDa}-\quad(E)$
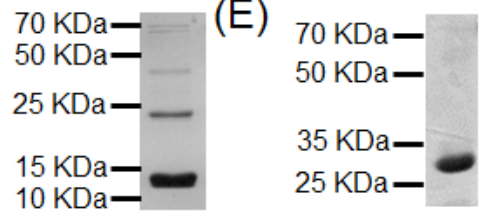

(F) Actin $(5 \mu \mathrm{M})++++++++-$
nPIST (CC1)- $-4 \mu \mathrm{M} 8 \mu \mathrm{M} 12 \mu \mathrm{M} 12 \mu \mathrm{M}$

(G) S P S PS PS P S P

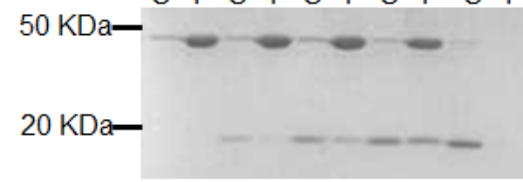
Actin $(5 \mu \mathrm{M})++++++++\ldots$ nPIST (CC2)- - $10 \mu \mathrm{M} 20 \mu \mathrm{M} 30 \mu \mathrm{M} 30 \mu \mathrm{M}$

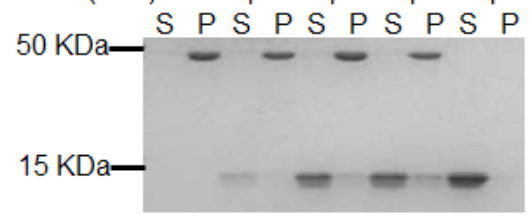

(H)

(I)

Actin $(5 \mu \mathrm{M})++++++++\ldots$ nPIST (WH2)- - $5 \mu \mathrm{M} 10 \mu \mathrm{M} 15 \mu \mathrm{M} 15 \mu \mathrm{M}$

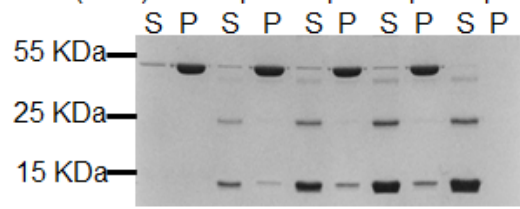

Actin $(5 \mu \mathrm{M})++++++++-$

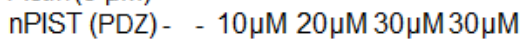

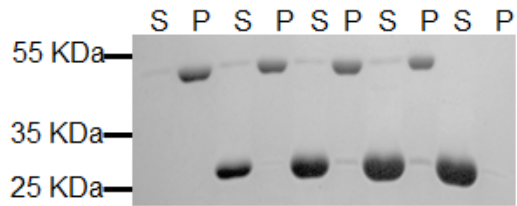


Figure 6:

(A)

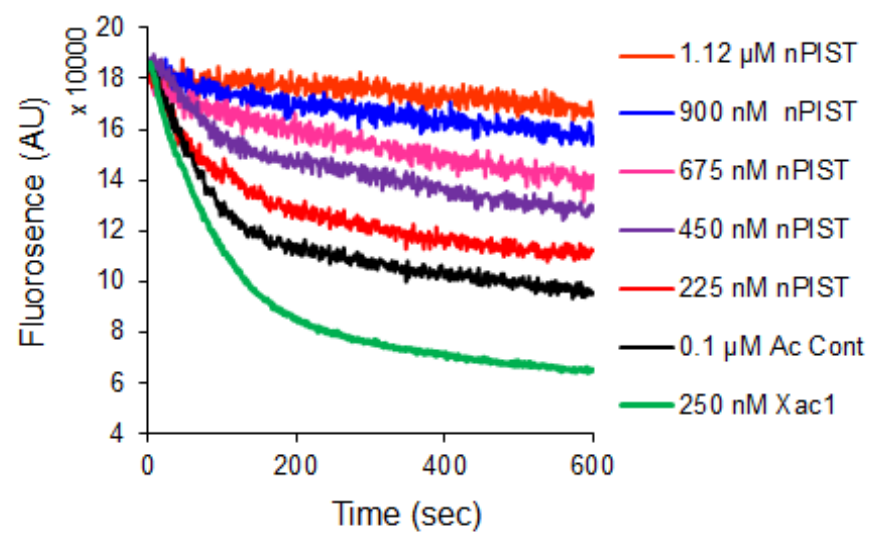

(B)

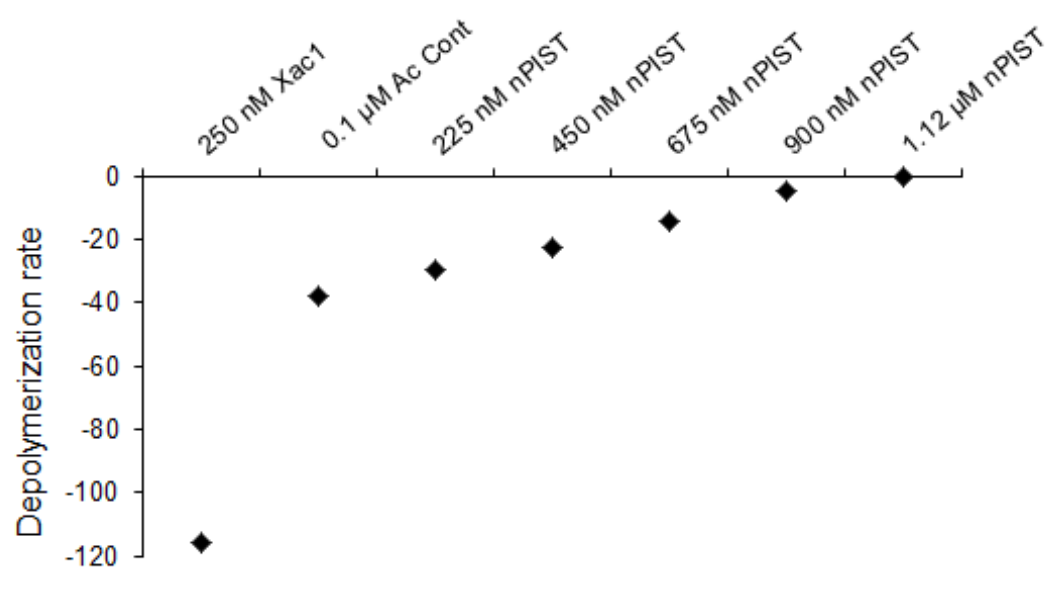


Figure 7:

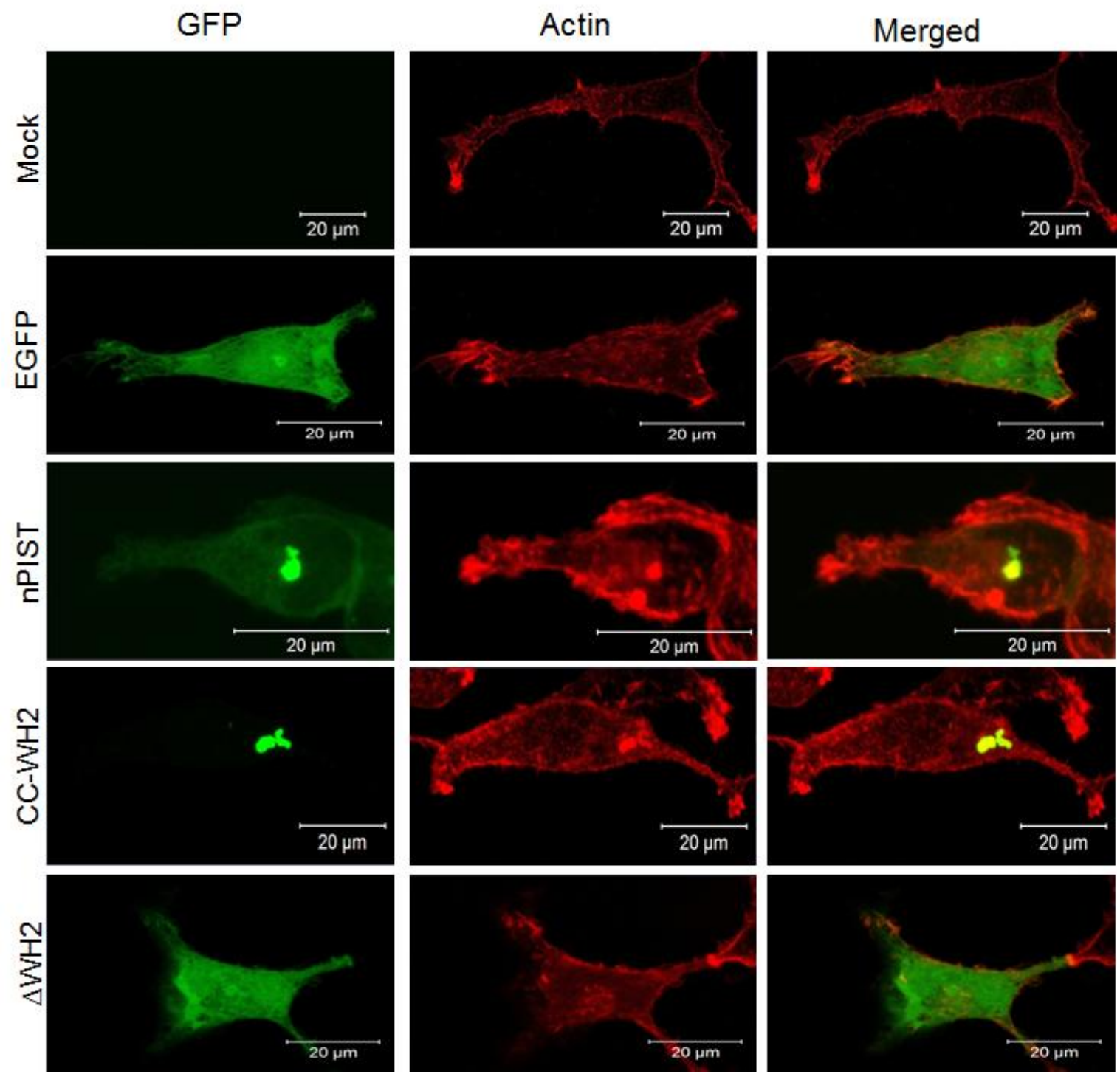


Figure S1:

(A)

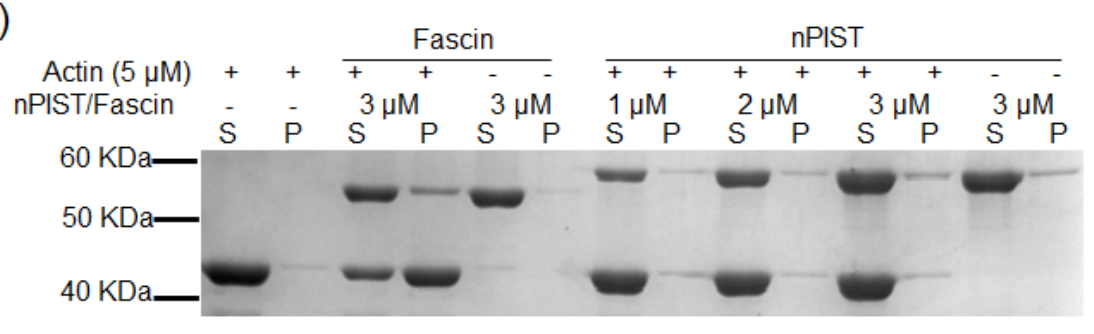

(B)

(C)
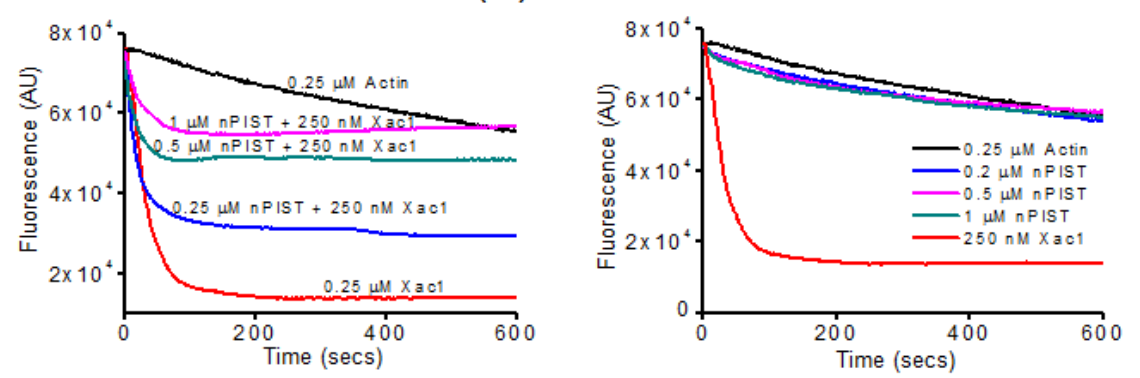

(D)

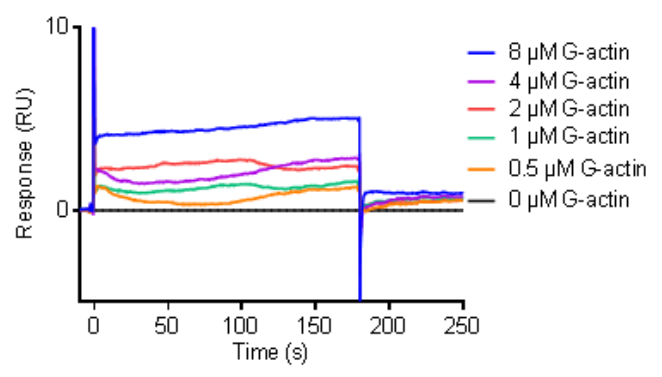


Table 1:

\begin{tabular}{|c|c|c|c|c|}
\hline Chain ID & Residue & Number & KFC2-A & KFC2-B \\
\hline B & ALA & 233 & ------- & ------- \\
\hline B & HIS & 234 & ------- & ------- \\
\hline B & ASP & 235 & ------- & ------- \\
\hline B & LEU & 237 & ------- & ------- \\
\hline B & TRP & 238 & Hotspot & Hotspot \\
\hline B & GLN & 240 & Hotspot & ------- \\
\hline B & LEU & 241 & Hotspot & Hotspot \\
\hline B & GLU & 242 & ------- & ------- \\
\hline B & GLU & 244 & ------- & ------- \\
\hline B & ILE & 245 & Hotspot & Hotspot \\
\hline B & HIS & 246 & ------- & ------- \\
\hline B & ARG & 249 & ------- & ------- \\
\hline
\end{tabular}

Table 2:

\begin{tabular}{|l|l|l|}
\hline$K_{\mathbf{D}}(\mathbf{M})$ & $\boldsymbol{k}_{\mathbf{a}}\left(\mathbf{M}^{-\mathbf{1}} \mathbf{s}^{-\mathbf{1}}\right)$ & $\boldsymbol{k}_{\mathbf{d}}\left(\mathbf{s}^{-\mathbf{1}}\right)$ \\
\hline $13.3 \times 10^{-9}$ & $9.25 \times 10^{4}$ & $1.23 \times 10^{-3}$ \\
\hline
\end{tabular}

